\title{
CARACTERIZACIÓN EN AMBIENTES BIOLÓGICOS DEL COMPORTAMIENTO A LA CORROSIÓN DE PELÍCULAS DELGADAS DE TI6AI4V CRECIDAS POR PULVERIZACIÓN CATÓDICA RF SOBRE SUSTRATOS DE ACERO 316L
}

Por:

E. C. Corredor ${ }^{1}$, C. A. Ortiz', E. Vera' ${ }^{1}$, Y J. E. Alfonso ${ }^{2}$

\section{RESUMEN}

En el presente trabajo se crecieron películas delgadas de Ti6Al4V sobre sustratos de acero 316L, con la técnica de pulverización catódica magnetrón rf, variando los parámetros de crecimiento. A los recubrimientos se les realizó un estudio morfológico y de composición a través de Microscopia Electrónica de Barrido (SEM) y análisis estructural mediante difracción de rayos $X(D R X)$ ). El comportamiento a la corrosión, se estableció por medio de la técnica de espectroscopia de impedancia electroquímica (EIS). Los resultados de SEM muestran que a medida que se incrementa la potencia incidente sobre el blanco la homogeneidad de las películas aumenta y la composición de la película no tiene una variación significativa. El estudio de DRX muestra que existe un crecimiento de los planos (1 10) y (220) de la fase $\beta$ de la aleación. Los resultados de EIS han permitido determinar que la resistencia a la corrosión se incrementa, pues el valor de la resistencia a la polarización de las probetas recubiertas se incrementa 3 órdenes de magnitud con respecto al sustrato.

Palabras Clave: Biomateriales; Películas Delgadas; Corrosión.

\section{ABSTRACT}

In the current Project some thin films of Ti6Al4V were grown on steel substrates $316 \mathrm{~L}$, with the technique of magnetron sputtering, varying the growth parameters. A morphologic and composition study was carried out to the coverings through a Scanning Electronic Microscopy (SEM) and a structural analysis by means or X-rays diffraction (XRD). The behavior to the corrosion was established by means of spectroscopy technique of electrochemical impedance (EIS). The SEM results shows that as the incident power is increased on the target the films have a higher density and the composition does not have a significant variation. The study of XRD shows that there is a growth of the planes (1 10) and (220) of the phase $\beta$ of the alloy. The EIS results have allowed determining that resistance to corrosion is increased to 3 orders of magnitude compared to that of the substrate.

Key words: Biomaterial; Thin Film; Corrosion.

\section{INTRODUCCIÓN}

Los biomateriales pueden ser considerados como productos aptos para ser utilizados en los seres humanos con fines de tratamiento o alivio de una enfermedad o lesión, o bien para la sustitución o modificación de su anatomía o de un proceso fisiológico (3). Entre sus características no puede faltar la de ser biocompatibles, que es la capacidad del biomaterial o dispositivo médico implantable para ser tolerado por el organismo o aceptado por el medio biológico que lo va a rodear, una vez implantado $(3,13)$. Otra propiedad que deben cumplir estos biomateriales es la de ser resistentes a la corrosión, ya que deben estar expuestos a un medio biológicamente agresivo como es el organismo humano. Dentro de los biomateriales metálicos mas utilizados en la actualidad se encuentran el acero inoxidable $316 \mathrm{~L}$ y las aleaciones de Titanio principalmente el Ti6Al4V $(3,13,11)$.

En este trabajo se depositó Ti6Al4V en forma de película delgada sobre sustratos de acero AISI 316L, mediante la técnica de sputtering magnetrón if. Las películas fueron caracterizadas estructuralmente mediante difracción de rayos $X(X R D)$, su composición y morfología fueron estudiadas mediante microscopia electrónica de barrido (SEM), y el desempeño ante la

\footnotetext{
'Universidad Pedagógica y Tecnológica de Colombia. Grupo de Superficies, Electroquímica y Corrosión (GSEC) ednaconsueloc@yahoo.es; cesaroti@tunja.uptc.edu.co; evera@tunja.uptc.edu.co.

2Universidad Nacional de Colombia. Grupo de Física de la Materia Condensada. Profesor Asociado al Centro Internacional de Física (CIF).
} 
Caracterización en ambientes biológicos del comportamiento a la corrosión de películas delgadas de Ti6Al4V crecidas por pulverización catódica RF sobre sustratos de acero $316 \mathrm{~L}$

corrosión con Espectroscopia de Impedancia Electroquímica (EIS).

\section{MATERIALES Y MÉTODOS}

Las probetas de acero $316 \mathrm{~L}$ fueron pulidas hasta lija 600, posteriormente pulidas en paño con alúmina hasta llegar a brillo espejo y finalmente, se les realizo limpieza con agua destilada, alcohol, acetona, alcohol, en su orden, por ultrasonido durante 10 minutos para cada caso.

El equipo utilizado para crecer las películas delgadas de Ti6Al4V es de la marca Alcatel modelo HS2000 ya referenciado en trabajos previos $(8,9)$. El sistema esta conformado por: un magnetrón rf; un sistema de vacío integrado por una cámara y bombas (mecánica y turbo molecular), un mezclador de gases, controles de flujo y presión final de gases. Las condiciones de trabajo en las que se lograron las películas fueron: presión total de $7 \times 10^{-3}$ mbar y $3 \times 10^{-3}$ mbar, flujo de gas Argón de 21.1 sccm, tiempo de depósito de 30 y 60 minutos. La potencia incidente sobre el blanco se vario entre 300 a 500W.

Figura 1. Esquema del equipo de pulverización catódica.

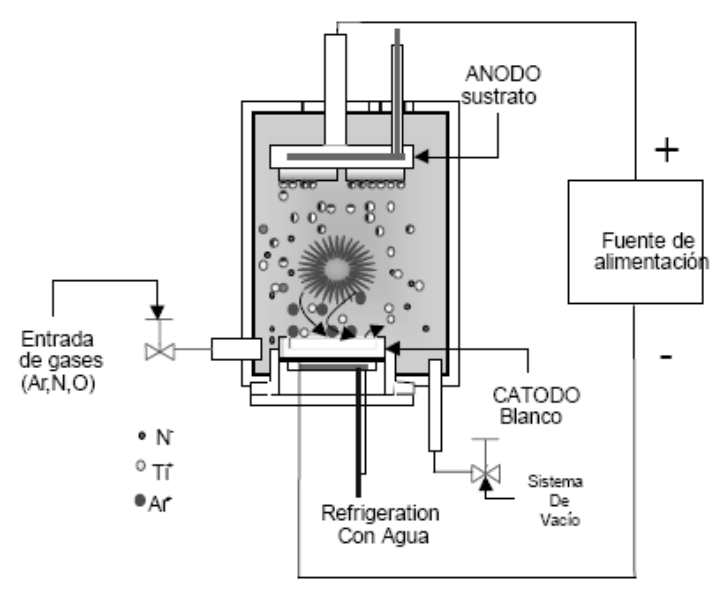

La caracterización de estos recubrimientos se llevo a cabo mediante, microscopia electrónica de
barrido(SEM), para obtener información como topografía, porosidad, utilizando un equipo de la marca LEO 430 con analizador EDXS; por otra parte, la caracterización estructural fue hecha mediante difracción de rayos $X(D R X)$ con un difractometro tipo PW1710 con la línea Ka del cobre, la corriente a través del tubo fue de $30 \mathrm{~mA}$ a una diferencia de potencial de $40 \mathrm{kv}$, y finalmente, para la evaluación electroquímica EIS se utilizo un potenciostato PC4 de Gamry Instruments. Se utilizó una celda plana, incorporando el electrodo de $\mathrm{Ag} / \mathrm{AgCl}$ como electrodo de referencia y un electrodo de platino, como contraelectrodo. La solución para realizar las pruebas fue $\mathrm{NaCl}$ al $1 \%$ con un $\mathrm{pH}$ de 7.4 y el rango de frecuencias utilizado fue de 10 $\mathrm{mHz}$ a $1 \mathrm{MHz}$.

\section{RESULTADOS Y DISCUSIÓN}

La figura 2 presenta los patrones de difracción (Intensidad $-2 \theta$ ) de las películas de Ti6Al4V crecidas sobre sustratos de acero $316 \mathrm{~L}$, a presiones de $7 \times 10^{-3}$ mbar y $3 \times 10^{-3}$ mbar, en función de la potencia incidente sobre el blanco. Los difractogramas dejan ver que en todos los casos se hacen presentes a $38.4^{\circ}$ los planos (110) y en algunos casos se revela el plano (220) a $82.4^{0}$ de la fase beta del titanio.

Los resultados de DRX determinan que el incremento de la potencia incidente sobre el blanco no tiene una influencia apreciable en el crecimiento de las películas, solo se puede establecer que a partir de $350 \mathrm{~W}$ se obtienen los planos (1 10) de la fase $\beta$ del Ti y que en algunos casos los planos (110) del material crecido se desplazan ligeramente hacia la izquierda. Este corrimiento, posiblemente, se pueda explicar en función de la tensión que existe entre la película y el sustrato debido a la diferencia de los coeficientes de expansión térmica entre el acero $316 \mathrm{~L}$ (16-18x10-6 $\left.\mathrm{K}^{-1}\right)$ (7) y el Ti6Al4V $\left(9.6 \times 10^{-6} \mathrm{~K}^{-1}\right)(10)$, ya que el aumento de la potencia hace que la temperatura del sustrato aumente, alcanzando temperaturas hasta de $400{ }^{\circ} \mathrm{C}$. 
Caracterización en ambientes biológicos del comportamiento a la corrosión de películas delgadas de Ti6Al4V crecidas por pulverización catódica RF sobre sustratos de acero $316 \mathrm{~L}$

Figura 2. Patrones de difracción de las películas de Ti6Al4V sobre sustratos de acero $316 \mathrm{~L}$. En ellos aparece el sustrato como referencia.

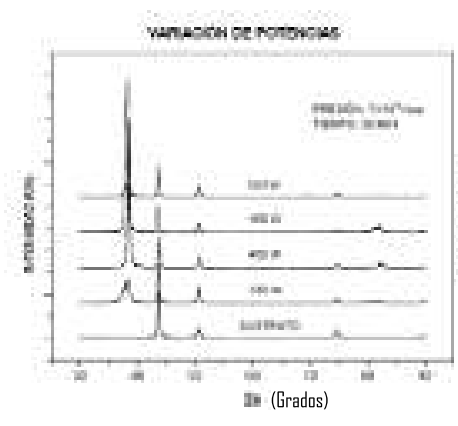

(a)

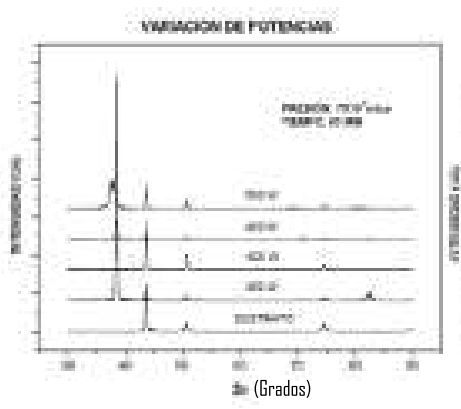

(c)

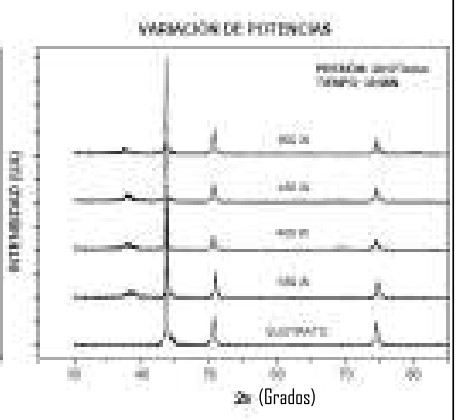

(b)

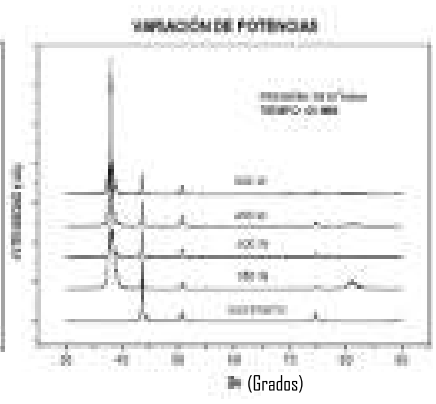

(d)
Por otra parte, los difractogramas de rayos $\mathrm{X}$ permiten establecer que las películas con mayor grado de cristalinidad se obtuvieron en un tiempo de una hora, con una presión de $7 \times 10^{-3}$ mbar y a una potencia de $350 \mathrm{~W}$ y además que las películas crecidas en media hora y a una presión de $3 \times 10^{-3}$ presentan baja cristalinidad y una película muy fina ya que los picos del Ti6Al4V tienen una intensidad relativa muy baja comparada con los picos del sustrato. Este resultado sugiere que el volumen de plasma que se tenga dentro de la cámara de depósito es importante en los procesos de crecimiento de películas que se obtengan a partir de blancos de composición compleja, ya que un plasma producido a mayor presión implica una mayor cantidad de especies ionizadas, lo que a su vez favorecerá la recombinación de estas especies sobre el sustrato produciendo un incremento en el espesor y calidad de la estructura cristalográfica de la película (4).

Figura 3. Micrografía de una película crecida con una potencia de $500 \mathrm{~W}$, presión de $7 \times 10^{-3}$ mbar y $30 \mathrm{~min}$ de depósito.

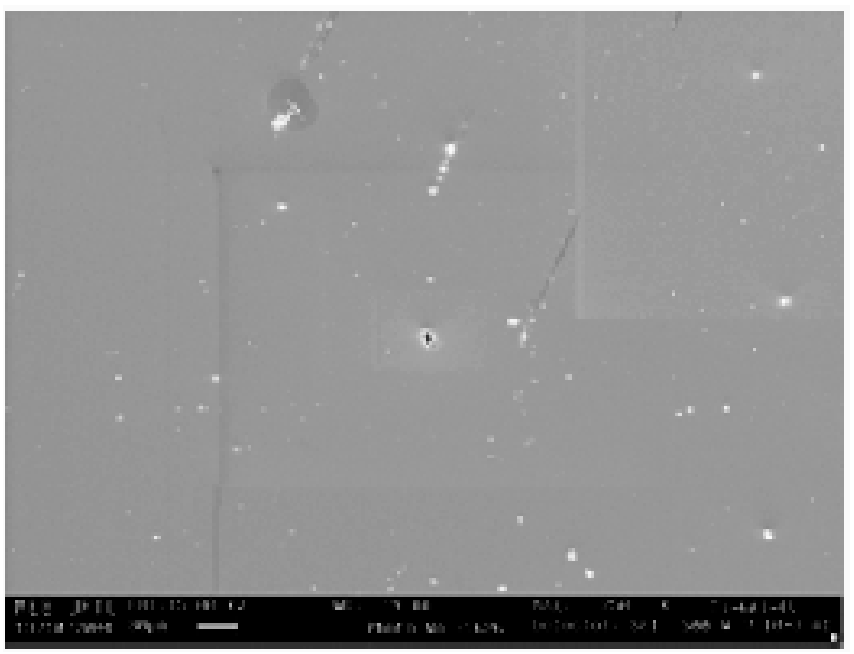

En la figura 3 se presenta una micrografía de una de las películas crecidas en condiciones experimentales anotadas. En la figura se puede apreciar que la película presenta un alto grado de homogeneidad, aunque existen partículas de material que no se han integrado completamente a la película, lo que indica que probablemente las condiciones termodinámicas no permitieron una completa coalescencia sobre la superficie del sustrato. Además, a pesar de que esta película se creció a una potencia de $500 \mathrm{~W}$, las películas en general, presentan una baja densidad de poros, lo que es un resultado positivo, ya que se espera que una película densa sea mas resistente a la corrosión.

Por otra parte, los resultados de EDXS obtenidos en los mismos recubrimientos permiten establecer que la composición de $\mathrm{Ti}, \mathrm{Al}$ y $\mathrm{V}$ no tiene una variación significativa, como se puede comprobar en la tabla 1. Los resultados se dan en porcentaje en peso con un margen de error de $3 \%$. 
Caracterización en ambientes biológicos del comportamiento a la corrosión de películas delgadas de Ti6Al4V crecidas por pulverización catódica RF sobre sustratos de acero $316 \mathrm{~L}$

Tabla1. Estudio cuantitativo elemental usando EDXS para algunas de las películas crecidas.

\begin{tabular}{|c|c|c|c|}
\hline MUESTRA & $\mathrm{Ti}$ & $\mathrm{Al}$ & $\mathrm{V}$ \\
\hline $350 \mathrm{~W}, 7 \times 10^{-3} \mathrm{mbar}, 30 \mathrm{~min}$ & 77.4 & 10.3 & 12.3 \\
\hline $400 \mathrm{~W}, 7 \times 10^{-3} \mathrm{mbar}, 30 \mathrm{~min}$ & 77.6 & 9.6 & 12.8 \\
\hline $500 \mathrm{~W}, 7 \times 10^{-3} \mathrm{mbar}, 30 \mathrm{~min}$ & 78.2 & 9.4 & 12.4 \\
\hline $350 \mathrm{~W}, 3 \times 10^{-3} \mathrm{mbar}, 30 \mathrm{~min}$ & 78.0 & 10.1 & 11.9 \\
\hline $400 \mathrm{~W}, 3 \times 10^{-3} \mathrm{mbar}, 30 \mathrm{~min}$ & 78.2 & 9.8 & 12.0 \\
\hline $500 \mathrm{~W}, 3 \times 10^{-3} \mathrm{mbar}, 30 \mathrm{~min}$ & 78.2 & 9.7 & 12.0 \\
\hline
\end{tabular}

En la figura 4. se muestra el diagrama de Bode obtenido para una muestra, cabe notar que el comportamiento es muy similar para todos los recubrimientos. Se observa claramente, que los recubrimientos aplicados incrementan notablemente el valor de la resistencia a la polarización $\left(R_{p}\right)$, respecto al acero sin recubrimiento (acero $316 \mathrm{~L})$. Una relación de estos valores permite determinar que la resistencia a la corrosión del material recubierto, se incrementa alrededor de tres órdenes de magnitud respecto al acero sin recubrimiento. Se observa además que los parámetros óptimos de deposición para obtener una película densa y de buen sellado, es de alrededor de 30 minutos. Se observa de los análisis de los diagramas de bode, que las películas ofrecen alta capacidad de protección al acero $316 \mathrm{~L}$, disminuyendo su tendencia a la generación de corrosión por picado en el mismo.

Los resultados obtenidos se modelaron mediante el circuito equivalente de la figura 5. el cual permitió el mejor ajuste, como se observa en la figura 4. Los valores obtenidos para los diferentes elementos del circuito se indican en la tabla 2.
Figura 4. Diagrama de Bode del sustrato y una película de Ti6Al4V crecida con una potencia de $450 \mathrm{~W}$, presión de $7 \times 10^{-3}$ mbar y tiempo de depósito de 60 minutos.
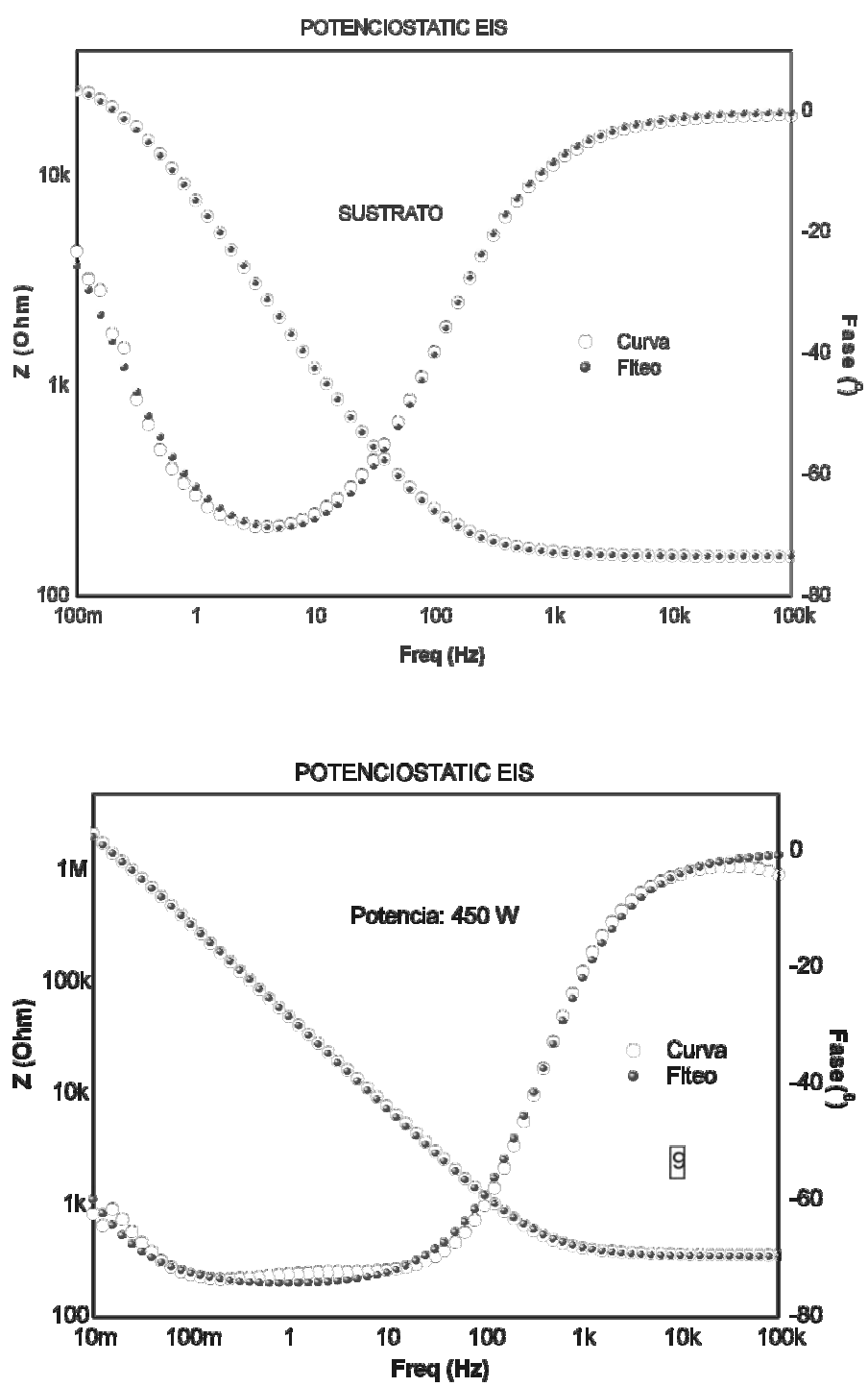

Figura 5. Circuito equivalente para las películas de Ti6Al4V sobre sustratos de acero $316 \mathrm{~L}$.

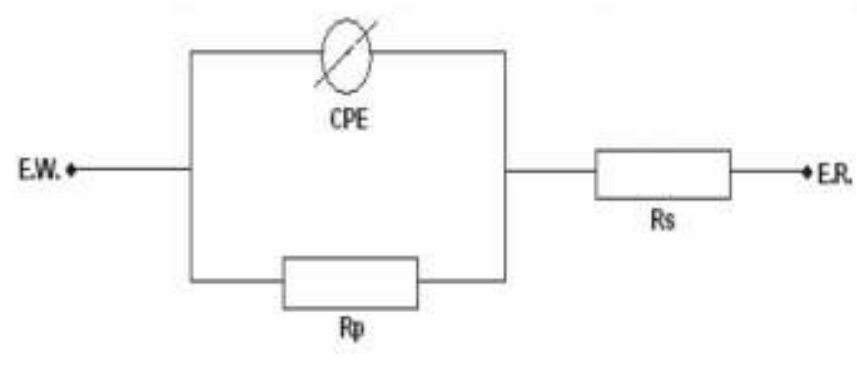


Caracterización en ambientes biológicos del comportamiento a la corrosión de películas delgadas de Ti6Al4V crecidas por pulverización catódica RF sobre sustratos de acero $316 \mathrm{~L}$

Tabla 2. Parámetros Calculados con el circuito equivalente.

\begin{tabular}{|c|c|c|c|c|c|}
\hline \multicolumn{2}{|c|}{ Muestra } & \multirow{2}{*}{$\frac{\operatorname{Rs}\left(\Omega_{1}\right)}{155.3}$} & \multirow{2}{*}{$\frac{\operatorname{CPE}\left(\mu_{f}\right)}{25.1}$} & \multirow{2}{*}{$\frac{\operatorname{Rp}(M \Omega)}{0.033}$} & \multirow{2}{*}{$\frac{\text { G.F.* }}{0.84}$} \\
\hline & sustrato & & & & \\
\hline \multirow{4}{*}{$\begin{array}{c}\mathrm{P}=3 \times 10^{-3} \\
\text { mbar } \\
\mathrm{T}=30 \text { minutos }\end{array}$} & $350 \mathrm{~W}$ & 175.1 & 6.67 & 11,14 & 0.88 \\
\hline & $400 \mathrm{~W}$ & 162.9 & 4.34 & 1,46 & 0.74 \\
\hline & $450 \mathrm{~W}$ & 160.8 & 5.85 & 7,5 & 0.82 \\
\hline & $500 \mathrm{~W}$ & 164.4 & 4.81 & 2,54 & 0.79 \\
\hline \multirow{4}{*}{$\begin{array}{c}\mathrm{P}=7 \times 10^{-3} \\
\text { mbar } \\
\mathrm{T}=30 \text { minutos }\end{array}$} & $350 \mathrm{~W}$ & 157.3 & 6.57 & 1,92 & 0.77 \\
\hline & $400 \mathrm{~W}$ & 169.8 & 2.27 & 2.10 & 0.82 \\
\hline & $450 \mathrm{~W}$ & 241.6 & 6.13 & 7.71 & 0.83 \\
\hline & $500 \mathrm{~W}$ & 89.4 & 14.94 & 2,09 & 0.90 \\
\hline \multirow{4}{*}{$\begin{array}{c}P=7 \times 10^{-3} \\
\text { mbar } \\
T=60 \text { minutos }\end{array}$} & $350 \mathrm{~W}$ & 295.4 & 5.36 & 6,9 & 0.88 \\
\hline & $400 \mathrm{~W}$ & 143.9 & 7.94 & 2,33 & 0.73 \\
\hline & $450 \mathrm{~W}$ & 253.4 & 12.36 & 0,92 & 0.83 \\
\hline & $500 \mathrm{~W}$ & 153.9 & 6.18 & 2.83 & 0.81 \\
\hline \multirow{4}{*}{$\begin{array}{c}\mathrm{P}=3 \times 10^{-3} \\
\text { mbar } \\
\mathrm{T}=60 \text { minutos }\end{array}$} & $350 \mathrm{~W}$ & 261 & 5.47 & 4,12 & 0.87 \\
\hline & $400 \mathrm{~W}$ & 153.2 & 9.07 & 0,97 & 0.79 \\
\hline & $450 \mathrm{~W}$ & 160.1 & 6.76 & 0,71 & 0.85 \\
\hline & $500 \mathrm{~W}$ & 158.2 & 4.37 & 4.92 & 0.85 \\
\hline
\end{tabular}

G.F.* Bondad de Fiteo.

\section{CONCLUSIONES}

En el presente trabajo se lograron obtener las mejores condiciones experimentales, para crecer películas delgadas de la fase $\beta$ del Ti, mediante la técnica magnetrón rf, con buena estructura cristalográfica y con una estequiometria aceptable.

Por otra parte, los resultados de EIS muestran que las películas con un alto grado de cristalinidad, presentan una resistencia a la polarización del orden de los $10^{7}$ ohmios, Si se tiene en cuenta, que la resistencia a la polarización del acero desnudo es del orden de $10^{4}$ ohmios, se puede concluir, que los recubrimientos ofrecen alta resistencia contra la corrosión.

\section{AGRADECIMIENTOS}

Los autores del presente trabajo expresan sus agradecimientos al CIF por prestar el equipo de pulverización catódica, al Doctor David Landinez por su colaboración en el análisis de resultados de DRX y a la Dirección de Investigaciones de la UPTC.

\section{BIBLIOGRAFÍA}

[1] BENTO, Carlos Alberto. Estudo da interface Ti-6Al4V/TiO2 por microscopia electrónica de barredura. Tesis de Maestría. Ciencia e Ingeniería de Materiales. (2000).

[2] Brauner, $\mathrm{H}$; Corrosión resístanse and biocompability of physical vapour deposition coatings for dental applications. Surface and coatings tecnology. 62, pp.618-625. (1993).

[3] CASTAÑEDA, Ernesto, Biomateriales de naturaleza inorgánica. Universidad Politécnica de Madrid. (2004).

[4] De la Rosa. Rubin, Franco. C, Valdes. E, Rodriguez. G. Dispersión Asistida por Magnetrón en Películas de TiN. Rev. Fac. Ing.-Univ. Tarapacá, vol. 13 No 2, 2oo5, pp. 31-38.

[5] Gamry instruments, Windows - based software getting started guide and primers and tutorials, Gamry Instruments, Inc. 2001, 3-2, 3-5.

[6] http://www.princetonapliedresearch.com/ applications/aplication_notes/078.pdf. Marzo (2005).

[7] http://www.goodfellow.com/csp/actie/STATIC/S/ Acero_Inoxidable_-_Inoxidable_-_AISI_316.HTML. Noviembre (2006).

[8] J.E. Alfonso, F. Pacheco, C. Moreno, R. Garzón, J. Torres. Influencia del voltaje de polarización del sustrato (bias), en la estructura cristalográfica de recubrimientos de Ti6Al4V, realizados mediante magnetrón if. Revista Colombiana de Física. Vol 35, No.1, 2003.

[9] J.E. Alfonso, F. Pacheco, C. Moreno, R. Garzón, J. Torres. Recubrimientos de TiN realizados mediante magnetrón rf. Revista Colombiana de Física. Vol.35, No 1, 2003. 
Caracterización en ambientes biológicos del comportamiento a la corrosión de películas delgadas de Ti6Al4V crecidas por pulverización catódica RF sobre sustratos de acero $316 \mathrm{~L}$

[10] J.M. Gómez-Vega, E. Saiz, A.P. Tomsia, G.W. Marshall, S.J. Marshall. A Multilayer Approach To Fabricate Bioactive Glass Coatings On Ti Alloys. http://www.osti.gov/bridge/servlets/purl/788020B8ngc6/native/788020.pdf. Noviembre (2006).

[11] ROJAS, Christian; LAGO, María E. Efectos de las proteínas del músculo esquelético en la corrosión de aceros inoxidables. Acta Científica Venezolana, 53: 156-163, 2002.

[12] RODRÍGUEZ, Daniel. Obtención de capas de nitruro de titanio mediante tratamiento termoquímico en Titanio y Ti6Al4V y caracterización de sus propiedades para aplicaciones biomédicas. Memoria de tesis para optar al grado de doctor ingeniero por la Universidad Politécnica Catalunya. (1999).

[13] VALLET, María Discurso. "Biomateriales: Repuesto para el cuerpo humano". A la Real Academia de Ingeniería. Madrid (2004).

Recibido: Septiembre 1 de 2006

Aceptado: Noviembre 30 de 2006 